\title{
Implementation of a generalized exponential basis functions method for linear and non-linear problems
}

\author{
F. Mossaiby ${ }^{1, *}$, M. Ghaderian ${ }^{1}$, R. Rossi ${ }^{2,3}$ \\ ${ }^{1}$ Department of Civil Engineering, University of Isfahan, 81744-73441 Isfahan, Iran \\ ${ }^{2}$ Centre Internacional de Mètodes Numèrics en Enginyeria (CIMNE), Barcelona, Spain \\ ${ }^{3}$ UPC, BarcelonaTech, Campus Norte UPC, 08034 Barcelona, Spain
}

\begin{abstract}
SUMMARY
In this paper, we address shortcomings of the method of exponential basis functions (EBF) by extending it to general linear and non-linear problems. In linear problems, the solution is approximated using a linear combination of exponential functions. The coefficients are calculated such that the homogenous form of equation is satisfied on some grid. To solve non-linear problems, they are converted to into a succession of linear ones using a Newton-Kantorovich approach. While the good characteristics of EBF are preserved, the generalized exponential basis functions method (GEBF) developed can be implemented with greater ease, as all calculations can be performed using real numbers and no characteristic equation is needed. The details of an optimized implementation are described. To study the performance of GEBF, we compare it on some benchmark problems with methods in the literature, such as variants of the boundary element method, where GEBF shows a good performance. Also in a 3D problem, we report the run time of the proposed method compared to Kratos, a parallel, highly optimized finite element code. The results show that to obtain the same level of error in the solution, much less computational effort and degrees of freedom is needed in the proposed method. Practical limits might be found however for large problems because of dense matrix operations involved. Copyright (c) 0000 John Wiley \& Sons, Ltd.
\end{abstract}

Received ...

KEY WORDS: Meshless methods; Exponential basis functions; Linear and non-linear problems; Partial differential equations; Newton-Kantorovich

\section{INTRODUCTION}

Meshless methods have received much attention from scientists and engineers in last decades. This can be related to difficulties of mesh-based methods due to efforts needed to create a suitable mesh. The development of a mesh generator program, especially for 3D problems, is a very delicate and time-consuming task. On the other hand, human expertise can never be completely eliminated from the process. From the early works on the smoothed particle hydrodynamics (SPH) [1,2] in 1977, there has been much progress in this regard. The element-free Galerkin method (EFG) [3], meshless

${ }^{*}$ Correspondence to: Department of Civil Engineering, University of Isfahan, 81744-73441 Isfahan, Iran

Copyright (C) 0000 John Wiley \& Sons, Ltd.

Prepared using nmeauth.cls [Version: 2010/05/13 v3.00] 
local Petrov-Galerkin (MLPG) [4], finite point method (FPM) [5-8], among others, can be named. Recently, methods based on the radial basis functions (RBFs) have been used by researchers to solve a wide range of problems [9-18]. The method of fundamental solutions (MFS), stemming from the boundary element method (BEM), is another method used successfully in a variety of problems [19-27]. Trefftz family of methods which try to approximate the solution using a Tcomplete set of basis functions have also been employed in many applications [28-35]. The main problem of MFS, BEM, and Trefftz family of methods is their dependency on fundamental solution and/or T-complete functions for the operator of interest. Obtaining such functions can be next to impossible in certain problem. Recently a Trefftz-like method was proposed by Boroomand and coworkers [36] which reduced the problem of obtaining T-complete like functions to solution of an algebraic equation. The main idea of this method is to use exponential basis functions where the exponents of the functions are chosen such that they satisfy the homogenous form of the differential equation, leading to an algebraic characteristic equation. The exponential basis function method (EBF) has been successfully applied in a wide range of problems, from heat conduction and elastic wave propagation to moving boundary problems and non-local elasticity [37-49].

The major limitation of EBF and other methods which rely upon it (like [50,51]), is that they can only solve problems with linear, constant-coefficient operators. While EBF has proved to perform very well in certain cases such as high-frequency problems, a wide range of popular problems, e.g. those involving materials with variable properties, cannot be handled. In this paper we generalize and extend the EBF method to linear problems with variable coefficients, as well as non-linear problems, using a Newton-Kantorovich scheme. Also, we drop completely the need for complex-valued calculations, even in wave propagation problems, which increases the simplicity and adoptability of the method. The formulation of the method in linear problems can be symbolically obtained from the one in $[52,53]$. The major difference is the use of exponential basis functions in a collocation approach, which eliminates the integrations in the former approach. This leads to simpler formulation and implementation. The method is then compared, in terms of errors and convergence rate to some of the methods found in the literature, like various BEM variants. To check the performance of the method with other well-established methods like the finite element method (FEM), we compare run time of the method with that of a parallel, highly optimized FEM code, Kratos [54] in a 3D problem, when both methods exhibit the same level of error. The comparison performed proves that, for the cases at hand, the computational cost needed to reach the same level of accuracy is much lower than for the FEM. One shall however acknowledge that the proposed method, similar to other alternatives of the same category, implies performing some time-consuming dense matrix operations. While such operations are very efficient and can be easily performed in parallel on commonplace or emerging hardware platforms such as CPUs and GPUs, their cost and memory requirements grows rapidly with the problem size. This implies that a practical limit might be found for very large problems. To show the possibilities of the method in problems with singularities, we solve the well-known Motz problem. We show that highly accurate results can be obtained by adding a few singular bases. This paves the road to solving 3D singular problems.

The structure of the paper is as follows: In the next section we review formulation of the EBF method. Afterwards, we present a generalized exponential basis function method (GEBF) for linear and non-linear problems. In Section 4 efficient implementation of the method is discussed. 
Numerical examples and comparison with other methods are presented in Section 5. Section 6 concludes the paper.

\section{BRIEF OVERVIEW OF THE EBF METHOD}

In this section we briefly review the EBF method for solving partial differential equations. Consider a $2 \mathrm{D}$ or $3 \mathrm{D}$ bounded domain $\Omega$ with boundary $\Gamma=\partial \Omega$. A general linear problem can be stated as

$$
\begin{aligned}
& \mathbf{L}_{\Omega} \mathbf{u}=\mathbf{f}_{\Omega} \quad \text { in } \Omega \\
& \mathbf{L}_{\Gamma} \mathbf{U}=\mathbf{f}_{\Gamma} \quad \text { on } \Gamma
\end{aligned}
$$

in which $\mathbf{u}$ is the vector of field variables and $\mathbf{L}_{\Omega}$ and $\mathbf{f}_{\Omega}$ represent, respectively, the linear differential operator and the specified right hand side function in $\Omega$. Also, $\mathbf{L}_{\Gamma}$ and $\mathbf{f}_{\Gamma}$ are the boundary operator and right hand side functions on $\Gamma$. In problems with mixed Dirichlet / Neumann boundaries, they take the form

$$
\mathbf{L}_{\Gamma}=\left\{\begin{array}{ll}
\mathbf{L}_{D} & \text { on } \Gamma_{D} \\
\mathbf{L}_{N} & \text { on } \Gamma_{N}
\end{array}, \quad \mathbf{f}_{\Gamma}= \begin{cases}\mathbf{f}_{D} & \text { on } \Gamma_{D} \\
\mathbf{f}_{N} & \text { on } \Gamma_{N}\end{cases}\right.
$$

where $\mathbf{L}_{D}$ and $\mathbf{L}_{N}$ represent, respectively, the Dirichlet and Neumann boundary operators. Also, $\Gamma_{D}$ and $\Gamma_{N}$ are Dirichlet and Neumann part of $\Gamma$ and $\mathbf{f}_{D}$ and $\mathbf{f}_{N}$ are defined respectively on them. The solution of (1) can be decomposed into a homogenous and a particular part as

$$
\mathbf{u}=\mathbf{u}^{h}+\mathbf{u}^{p}
$$

where $\mathbf{u}^{h}$ and $\mathbf{u}^{p}$ are chosen such that

$$
\begin{aligned}
\mathbf{L}_{\Omega} \mathbf{u}^{h} & =\mathbf{0} \\
\mathbf{L}_{\Omega} \mathbf{u}^{p} & =\mathbf{f}_{\Omega}
\end{aligned}
$$

From the linearity of $\mathbf{L}_{\Gamma}$ one may conclude that

$$
\mathbf{L}_{\Gamma} \mathbf{u}=\mathbf{L}_{\Gamma}\left(\mathbf{u}^{h}+\mathbf{u}^{p}\right)=\mathbf{L}_{\Gamma} \mathbf{u}^{h}+\mathbf{L}_{\Gamma} \mathbf{u}^{p}=\mathbf{f}_{\Gamma}
$$

For brevity, we consider only the case $\mathbf{f}_{\Omega}=\mathbf{0}$, in which we may take $\mathbf{u}^{p}=\mathbf{0}$. The general case can be found in [36] and is similar to the way we obtain the particular solution in the proposed method. The homogeneous part of the solution may be assumed as

$$
\mathbf{u}^{h} \approx \hat{\mathbf{u}}^{h}=\sum_{i=1}^{m^{h}} \boldsymbol{\Psi}_{i}^{h} c_{i}^{h}=\boldsymbol{\Psi}^{h} \mathbf{c}^{h}
$$

in which $m^{h}$ is the number of bases, $\Psi^{h}$ contains the exponential basis functions and $\mathbf{c}^{h}$ contains the respective coefficients. For example, in $2 \mathrm{D}$ problems these bases functions take the form of $\exp \left(\alpha_{i} x+\beta_{i} y\right)$ where $\alpha_{i}$ and $\beta_{i}$ can take on complex values, i.e. $\alpha_{i}, \beta_{i} \in \mathbb{C}$. In EBF, $\Psi^{h}$ is chosen such that it satisfies the homogenous governing partial differential equation. Substitution of the (6) 
in (4a) results in the following relation

$$
\mathbf{L}_{\Omega} \hat{\mathbf{u}}^{h}=\mathbf{L}_{\Omega} \Psi^{h} \mathbf{c}^{h}=\mathbf{H} \Psi^{h} \mathbf{c}^{h}=\mathbf{0}
$$

In order to obtain a non-trivial solution for the above homogenous equation, the determinant of $\mathbf{H}$ must vanish

$$
\operatorname{det} \mathbf{H}=0
$$

The above equation is referred to as the characteristic equation for the desired domain operator and plays an important role in the EBF method. From the above characteristic equation one may find, for example, $\alpha_{i}$ in terms of $\beta_{i}$ or vice versa. The reader may note that the equation (7) is only valid when $\mathbf{L}_{\Omega}$ is a linear operator with constant coefficients. The characteristic equation (8) is an algebraic equation, and it can be solved, analytically or numerically, for all constant coefficients operators. The characteristic equations for the solution of a variety of engineering problems can be found in the recent papers $[40,42,43,45-49]$.

\section{Remark 1}

The EBF method can be regarded as a generalization of the solution method used for constant coefficient ordinary differential equations. The term 'characteristic equation' is used with the same meaning in both contexts. The EBF method leads to exact solution in homogenous one-dimensional cases.

\section{THE PROPOSED METHOD}

In this section we present generalization of the EBF method. We will first describe the formulation of the proposed method for solving linear problems. Then we will employ an iterative scheme to solve non-linear problems.

\subsection{Linear problems}

Starting again from (1), we approximate $\mathbf{u}$ with a linear combination of basis functions as

$$
\mathbf{u}=\mathbf{u}^{h}+\mathbf{u}^{p} \approx \hat{\mathbf{u}}=\hat{\mathbf{u}}^{h}+\hat{\mathbf{u}}^{p}=\sum_{i=1}^{m} \boldsymbol{\psi}_{i} c_{i}=\sum_{i=1}^{m} \boldsymbol{\psi}_{i}\left(c_{i}^{h}+c_{i}^{p}\right)
$$

in which $m$ is the number of bases used. In matrix notation, (9) can be written as

$$
\mathbf{u} \approx \hat{\mathbf{u}}=\boldsymbol{\Psi} \mathbf{c}=\Psi\left(\mathbf{c}^{h}+\mathbf{c}^{p}\right)
$$

The particular part can be calculated as in [36]. To this end, a series of points, $\mathbf{x}_{\Omega, j}, j=1, \ldots, n_{\Omega}$ are chosen in the solution domain, $\Omega$. Then (4b) is applied in these points as

$$
\left.\mathbf{L}_{\Omega} \hat{\mathbf{u}}^{p}\right|_{\mathbf{x}_{\Omega, j}}=\left.\mathbf{L}_{\Omega} \Psi\right|_{\mathbf{x}_{\Omega, j}} \mathbf{c}^{p}=\left.\mathbf{f}_{\Omega}\right|_{\mathbf{x}_{\Omega, j}}
$$


In matrix notation, (11) can be written as

$$
\mathbf{Q} \mathbf{c}^{p}=\mathbf{h}
$$

in which $j$-th row of $\mathbf{Q}$ and $\mathbf{h}$ are defined as

$$
\begin{gathered}
(\mathbf{Q})_{j}=\left.\mathbf{L}_{\Omega} \Psi\right|_{\mathbf{x}_{\Omega, j}} \\
(\mathbf{h})_{j}=\left.\mathbf{f}_{\Omega}\right|_{\mathbf{x}_{\Omega, j}}
\end{gathered}
$$

From (12) the coefficients $\mathbf{c}^{p}$ can be calculated as

$$
\mathbf{c}^{p}=\mathbf{Q}^{+} \mathbf{h}
$$

where the ' + ' superscript denotes the Moore-Penrose generalized inverse.

\section{Remark 2}

The generalized inverse of $\mathbf{Q}$ is not formed explicitly. Instead, the singular value decomposition (SVD) of the matrix, computed using LAPACK library is employed. More details on the implementation will be presented in Section 4.

From the above equation, $\hat{\mathbf{u}}^{p}$ can be calculated as

$$
\hat{\mathbf{u}}^{p}=\boldsymbol{\Psi} \mathbf{c}^{p}=\boldsymbol{\Psi} \mathbf{Q}^{+} \mathbf{h}
$$

Applying (4a) in $\mathbf{x}_{\Omega, j}$ one may conclude

$$
\left.\mathbf{L}_{\Omega} \hat{\mathbf{u}}^{h}\right|_{\mathbf{x}_{\Omega, j}}=\left.\mathbf{L}_{\Omega} \Psi\right|_{\mathbf{x}_{\Omega, j}} \mathbf{c}^{h}=\mathbf{0}
$$

or, in matrix notation

$$
\mathbf{Q c}^{h}=\mathbf{0}
$$

where $\mathbf{Q}$ is defined in (13a). For a non-trivial solution, $\mathbf{c}^{h}$ must be in the null space of the matrix $\mathbf{Q}$

$$
\mathbf{c}^{h} \in \operatorname{null}(\mathbf{Q})
$$

More details on the subject of calculating the null space of $\mathbf{Q}$ will be presented in Section 4. If $\mathbf{c}^{h}$ satisfies the above equation, it can be written as a linear combination of the bases of the space, namely $\mathbf{t}_{i}$

$$
\mathbf{c}^{h}=\sum_{i=1}^{b} \mathbf{t}_{i} d_{i}=\mathbf{T d}
$$

where $d_{i}$ are unknown coefficients, $b$ is the number of bases spanning the space, and $\mathbf{T}$ is a matrix with its columns being the bases, $\mathbf{t}_{i}$.

\section{Remark 3}


The null space bases are calculated from the SVD of the matrix $\mathbf{Q}$ used previously in equation (14) as will be discussed in Section 4.

Inserting (19) into (10) one obtains

$$
\hat{\mathbf{u}}^{h}=\boldsymbol{\Psi} \mathbf{T d}
$$

To apply the boundary conditions, a series of points, $\mathbf{x}_{\Gamma, j}, j=1, \ldots, n_{\Gamma}$ are chosen on the boundary and a collocation approach is used. From (5) one may conclude that

$$
\left.\mathbf{L}_{\Gamma} \hat{\mathbf{u}}^{h}\right|_{\mathbf{x}_{\Gamma, j}}=\left.\mathbf{f}_{\Gamma}\right|_{\mathbf{x}_{\Gamma, j}}-\left.\mathbf{L}_{\Gamma} \hat{\mathbf{u}}^{p}\right|_{\mathbf{x}_{\Gamma, j}}
$$

Combining (15) and (20), the above equation can be written as

$$
\left.\mathbf{L}_{\Gamma} \Psi\right|_{\mathbf{x}_{\Gamma, j}} \mathbf{T d}=\left.\mathbf{f}_{\Gamma}\right|_{\mathbf{x}_{\Gamma, j}}-\left.\mathbf{L}_{\Gamma} \Psi\right|_{\mathbf{x}_{\Gamma, j}} \mathbf{c}^{p}
$$

from which $\mathbf{d}$ can be obtained as

$$
\mathbf{d}=(\mathbf{P T})^{+}\left(\mathbf{g}-\mathbf{P c}^{p}\right)
$$

In the above equation, $j$-th row of $\mathbf{P}$ and $\mathbf{g}$ are defined as

$$
\begin{aligned}
(\mathbf{P})_{j} & =\left.\mathbf{L}_{\Gamma} \Psi\right|_{\mathbf{x}_{\Gamma, j}} \\
(\mathbf{g})_{j} & =\left.\mathbf{f}_{\Gamma}\right|_{\mathbf{x}_{\Gamma, j}}
\end{aligned}
$$

\section{Remark 4}

Equation (23) is mathematically equivalent to a linear least squares problem, and can be solved with a single call to LAPACK function, DGELSD. More details will follow in Section 4.

\section{Remark 5}

As can be seen, the formulation is general in the sense that any kind of basis functions, $\Psi$, could be used. However, we choose the exponential basis functions as used in EBF. This has proved to be very efficient in terms of approximation capabilities in smooth problems. As the bases no longer have the constraint of satisfying the homogenous form of the differential equation, we use real and imaginary parts of the aforementioned functions separately, so that all of the calculations can be performed using real numbers. Consider for example an exponential basis function in a 3D scalar problem as

$$
\psi=\exp (\alpha x+\beta y+\gamma z), \quad \alpha=a+\mathrm{i} b, \beta=c+\mathrm{i} d, \gamma=e+\mathrm{i} f, \quad a, b, c, d, e, f \in \mathbb{R}
$$

We use the real and imaginary parts of it as

$$
\begin{aligned}
& \psi_{1}=\mathfrak{R}(\psi)=\exp (a x+c y+e z) \cos (b x+d y+f z) \\
& \psi_{2}=\mathfrak{I}(\psi)=\exp (a x+c y+e z) \sin (b x+d y+f z)
\end{aligned}
$$


as one of the basis functions in (9). In 3D examples we take $a, b, c, d, e, f \in\{-1,0,+1\}$ or $\{-1,-1 / 3,+1 / 3,+1\}$ for 1458 and 8192 basis functions respectively.

\section{Remark 6}

For problems with non-smooth or high-gradient solution, usual approaches can be employed. One approach can be a domain-decomposition scheme which, if used correctly, can reduce the effects of the singularities (see for example [55]). Reducing the size of sub-domains leads to a local form of the proposed method. In this form the domain is discretized into 'clouds', similar to conventional meshless methods. Each cloud is treated as a single domain formulated using the proposed method. All resulting equations are solved together in a system of equations. This approach has been employed in [50,51], implementing a local form of EBF. In this case the final coefficient matrix will be sparse, and could be solved using various available techniques.

On the other hand, one may employ specially crafted functions with appropriate singularity shape and position. These functions absorb the effects of the singularities and leave the (rather) smooth parts to exponential basis functions. Such bases has been used in a variety of methods, such as collocation Trefftz methods [56-58] and EBF [59]. We employ this approach in Section 5 to solve a singular problem.

By obtaining $\mathbf{d}$ (and hence $\mathbf{c}^{h}$ ) and $\mathbf{c}^{p}$, the unknown function $\mathbf{u}$ can be computed in any desired location in the domain.

\subsection{Non-linear problems}

To extend the proposed method to non-linear problems, we employ a Newton-Kantorovich (NK) [60] scheme to linearize the PDE. The resulting linear equations are solved using the proposed method in Section 3.1 in an iterative manner. To this end, consider a general non-linear problem as

$$
\begin{array}{ll}
\mathbf{N}_{\Omega} \mathbf{u}=\mathbf{f}_{\Omega} & \text { in } \Omega \\
\mathbf{N}_{\Gamma} \mathbf{u}=\mathbf{f}_{\Gamma} \quad \text { on } \Gamma
\end{array}
$$

where $\mathbf{N}_{\Omega}$ and $\mathbf{N}_{\Gamma}$ represent non-linear operators on the domain and boundary, respectively. To satisfy the above equation, we may set the appropriate residuals in the domain and on the boundary to zero

$$
\begin{aligned}
& \mathbf{r}_{\Omega}=\mathbf{N}_{\Omega} \mathbf{u}-\mathbf{f}_{\Omega} \\
& \mathbf{r}_{\Gamma}=\mathbf{N}_{\Gamma} \mathbf{u}-\mathbf{f}_{\Gamma}
\end{aligned}
$$

Taking variation from the above equations, one gets

$$
\begin{gathered}
\delta \mathbf{r}_{\Omega}=\delta\left(\mathbf{N}_{\Omega} \mathbf{u}-\mathbf{f}_{\Omega}\right)=\delta\left(\mathbf{N}_{\Omega} \mathbf{u}\right)=\overline{\mathbf{L}}_{\Omega} \delta \mathbf{u} \\
\delta \mathbf{r}_{\Gamma}=\delta\left(\mathbf{N}_{\Gamma} \mathbf{u}-\mathbf{f}_{\Gamma}\right)=\delta\left(\mathbf{N}_{\Gamma} \mathbf{u}\right)=\overline{\mathbf{L}}_{\Gamma} \delta \mathbf{u}
\end{gathered}
$$


where $\overline{\mathbf{L}}_{\Omega}$ and $\overline{\mathbf{L}}_{\Gamma}$ are the so called Fréchet derivative operators and represent respectively the linearized operators on the domain and boundary. The residuals and the unknown function $\mathbf{u}$ can be approximated as

$$
\mathbf{u}^{(k+1)}=\mathbf{u}^{(k)}+\delta \mathbf{u}^{(k)}
$$

and

$$
\begin{aligned}
& \mathbf{r}_{\Omega}^{(k+1)}=\mathbf{r}_{\Omega}^{(k)}+\delta \mathbf{r}_{\Omega}^{(k)} \\
& \mathbf{r}_{\Gamma}^{(k+1)}=\mathbf{r}_{\Gamma}^{(k)}+\delta \mathbf{r}_{\Gamma}^{(k)}
\end{aligned}
$$

Setting the residuals in $(k+1)$-th step to zero will yield to

$$
\begin{aligned}
& \delta \mathbf{r}_{\Omega}^{(k)}=-\mathbf{r}_{\Omega}^{(k)} \\
& \delta \mathbf{r}_{\Gamma}^{(k)}=-\mathbf{r}_{\Gamma}^{(k)}
\end{aligned}
$$

or equivalently

$$
\begin{aligned}
\overline{\mathbf{L}}_{\Omega}^{(k)} \delta \mathbf{u}^{(k)} & =-\mathbf{r}_{\Omega}^{(k)} \\
\overline{\mathbf{L}}_{\Gamma}^{(k)} \delta \mathbf{u}^{(k)} & =-\mathbf{r}_{\Gamma}^{(k)}
\end{aligned}
$$

The operators depend on the unknown function in the $(k)$-th step, i.e. $\mathbf{u}^{(k)}$. Equations (33) are linear equations which may be solved using the aforementioned method to obtain $\delta \mathbf{u}^{(k)}$ and update $\mathbf{u}$ in equation (30). The reader may note that

$$
\delta \hat{\mathbf{u}}=\delta \hat{\mathbf{u}}^{h}+\delta \hat{\mathbf{u}}^{p}=\boldsymbol{\Psi} \delta \mathbf{c}=\boldsymbol{\Psi}\left(\delta \mathbf{c}^{h}+\delta \mathbf{c}^{p}\right)
$$

Hence, one only needs to obtain $\delta \mathbf{c}^{h}$ and $\delta \mathbf{c}^{p}$ and update $\mathbf{c}^{h}$ and $\mathbf{c}^{p}$ respectively.

\section{Remark 7}

The iteration scheme described above resembles the Newton-Raphson method in algebraic equations. Other variations like the modified Newton-Kantorovich method (MNK) are also available and could be employed; see [60] for more information. We will discuss this in more detail in the numerical examples section.

\section{Remark 8}

The linearization approach described is quite general, and can be used to solve a wide class of non-linear problems, including large deformation and materially non-linear problems. For example, in case of large deformation problems, a Total Lagrangian scheme can be used. To do so, one may start by linearizing the equilibrium equations in terms of second Piola-Kirchhof (PK2) stresses and proceed as usual to formulate the linearized equations in terms of variations of displacements. We aim to present this specific case in a separate study. 


\section{IMPLEMENTATION}

Calculating the null space bases of $\mathbf{Q}$, as well as the calculation of the coefficients for homogenous and particular parts of the solution are the most important parts of the solution procedure. These parts take the most of execution time, and hence, should be carefully implemented. Other optimizations, like parallelizing the construction of $\mathbf{Q}$, are possible and will be discussed.

\subsection{Efficient implementation of the proposed method}

A brief look at the formulation presented in Section 3 reveals that both the Moore-Penrose generalized inverse and null space bases of $\mathbf{Q}$ are required. The most robust way to calculate both of these for a matrix is known to be the SVD. The SVD of a given matrix $\mathbf{Q}$ can be written as

$$
\mathbf{Q}=\mathbf{U} \Sigma \mathbf{V}^{*}
$$

in which $\mathbf{U}$ and $\mathbf{V}$ are called left and right singular vectors, and $\boldsymbol{\Sigma}$ is a diagonal matrix, containing the singular values, $\sigma_{k}$, i.e.,

$$
(\boldsymbol{\Sigma})_{k k}=\sigma_{k}, \quad \text { no sum on } k
$$

The superscript ' $*$ ' stands for conjugate-transpose of a matrix. The Moore-Penrose generalized inverse (or pseudo-inverse) of a matrix is defined as [61]

$$
\mathbf{Q}^{+}=\mathbf{V} \boldsymbol{\Sigma}^{+} \mathbf{U}^{*}
$$

where the diagonal matrix $\boldsymbol{\Sigma}^{+}$is defined as

$$
\left(\boldsymbol{\Sigma}^{+}\right)_{k k}=\left\{\begin{array}{ll}
\left(\sigma_{k}\right)^{-1} & \sigma_{k} \neq 0 \\
0 & \sigma_{k}=0
\end{array}, \quad \text { no sum on } k\right.
$$

The columns of $\mathbf{V}$ where the corresponding singular value are zero have no effect in the construction of $\mathbf{Q}^{+}$; on the other hand, these columns form the null space bases of $\mathbf{Q}$. Hence, by calculating the SVD of $\mathbf{Q}$, both its pseudo-inverse and null space bases can be robustly calculated. We use this point to decrease the needed calculations. One may also note that as we only need the product of $\mathbf{Q}^{+}$with a vector, we can avoid forming $\mathbf{Q}^{+}$explicitly, and do the calculations such that we always have a matrix-vector product. This not only decreases greatly the time required to do the calculations, but also reduces the round-off errors.

The SVD was calculated unsing the DGESDD function of LAPACK [62] library. To obtain high performance in this step, LAPACK was used along with OpenBLAS [63]. This paved the road to harness the power of modern multi-core CPUs. OpenBLAS is an optimized parallel implementation of the BLAS [64] which is freely available. Both LAPACK and BLAS are well established libraries, and many vendors provide optimized versions of them along with their hardware.

The product of the pseudo-inverse of a matrix with a vector is equivalent to a linear least-squares problem, e.g.

$$
\mathbf{x}=\mathbf{A}^{+} \mathbf{b} \Leftrightarrow\|\mathbf{A} \mathbf{x}-\mathbf{b}\|_{2}=\min .
$$


If the null space bases of a matrix is not needed, such as the case in (23), this can be readily done with LAPACK function, DGELSD. This is slightly faster than calculating a truncated SVD and performing the multiplication. The configuration described above ensures that high performance can be obtained using this method.

\section{Remark 9}

As we use standard LAPACK functions, leveraging the code to benefit from other high performance LAPACK implementations seems to be possible. As an example, one might try to employ emerging high performance platforms such as GPUs. This may be important recalling the fact that the matrices used in the proposed method are dense. These type of matrices are very suitable for GPU platform, and the calculations can be done much faster on them. There are already several LAPACK implementations available for this purpose, however, investigation of performance of the proposed method on such platform is beyond the scope of this work.

The construction of $\mathbf{Q}$ offers complete granularity, and hence, is a good candidate for parallelization. Here we used OpenMP to achieve a good speedup in this operation. The parallelization can be easily performed in FORTRAN or $\mathrm{C} / \mathrm{C}++$, just by adding appropriate statements in the code that instructs the compiler to generate appropriate parallel code. Extra care should be taken to avoid race-condition scenarios. The complete source code of the implementation used can be accessed freely at BitBucket [65].

\subsection{Step-by-step procedure of the proposed method}

The steps needed to solve a linear problem using the proposed method are summarized below.

1. Build the matrix $\mathbf{Q}$ using (13a)

2. Obtain the SVD of matrix $\mathbf{Q}$

3. Calculate $\mathbf{c}^{p}$ from (14)

4. Calculate $\mathbf{c}^{h}$ from (19) by first obtaining $\mathbf{d}$ using (23)

5. Form the final solution $\hat{\mathbf{u}}$

In case of non-linear problems, the following approach could be used.

1. Linearize the equation and boundary conditions using (29)

2. Consider an initial guess for $\mathbf{c}^{h}$ and $\mathbf{c}^{p}$; use $\mathbf{0}$ if no better information is available, e.g. from a previous increment in an incremental solution

3. Solve (33) for $\delta \mathbf{c}^{h}$ and $\delta \mathbf{c}^{p}$ using the method described for linear equations

4. Update $\mathbf{c}$ using (30) and $\delta \mathbf{c}^{h}$ and $\delta \mathbf{c}^{p}$ calculated above

5. While some convergence criteria are not met, go to Step 3

If the MNK method is to be used, in Step 3 one can use the SVD calculated in a previous step to decrease the computational cost. In highly non-linear problems, calculating the SVD every few iterations might help convergence. 


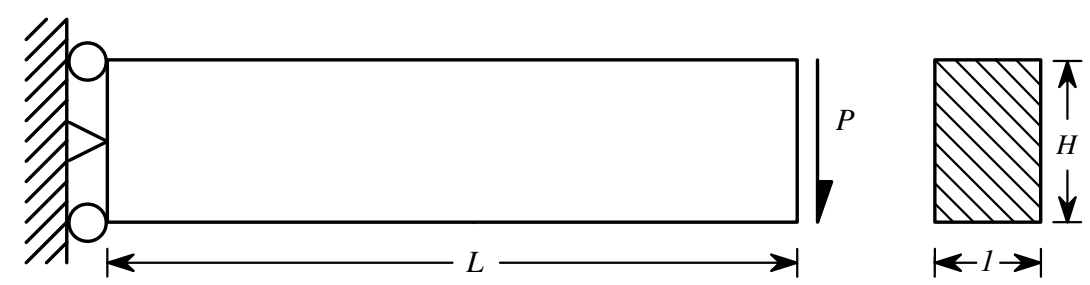

Figure 1. Cantilever beam under end load

\section{NUMERICAL EXAMPLES}

In this section we present some numerical examples to demonstrate the capability of the proposed method in the solution of a range of problems. To report the deviation of the numerical solutions from the exact solutions, we choose the $L_{2}$ error norm as

$$
e_{L_{2}}=\sqrt{\frac{\sum_{i=1}^{n}\left[(\hat{\phi})_{i}-(\phi)_{i}\right]^{2}}{\sum_{i=1}^{n}(\phi)_{i}^{2}}}
$$

where $n$ is the number of points selected inside the domain and on its boundaries for calculation of the errors. Also $(\hat{\phi})_{i}$ and $(\phi)_{i}$ are the approximate and exact solutions at the $i$-th selected point, respectively.

\section{Example 1}

As the first example, we aim to solve a two dimensional cantilever beam problem. Consider a beam of length $L$ and height $H$ subjected to traction at the free end (viz. Figure 1). The beam has a unit thickness and hence a plane stress problem is considered. The exact solution can be found in [66] as

$$
\begin{aligned}
& u_{x}=\frac{P y}{6 E I}\left[x(6 L-3 x)+(2+v)\left(y^{2}-\frac{H^{2}}{4}\right)\right] \\
& u_{y}=-\frac{P}{6 E I}\left[3 v y^{2}(L-x)+(4+5 v) \frac{H^{2} x}{4}+x^{2}(3 L-x)\right]
\end{aligned}
$$

where $u_{x}$ and $u_{y}$ are displacement components along $x$ and $y$ directions, $v$ is the Poisson ratio, $E$ is the elasticity modulus and $I$ is the moment of inertia of the beam given by $I=H^{3} / 12$. Also the stresses corresponding to the above displacements are

$$
\sigma_{x}=\frac{P y(L-x)}{I}, \quad \sigma_{y}=0, \quad \tau_{x y}=-\frac{P}{2 I}\left(\frac{H^{2}}{4}-y^{2}\right)
$$

In this example we use $E=3.0 \times 10^{7}, v=0.3, H=12, L=48$ and $P=1000$. The conditions at the boundary points are as shown in the figure. Figure 2 shows the $L_{2}$ error norm for displacements and stresses versus the average distance between nodes, $h$, in logarithmic scale. Figure 3 shows a comparison between the exact solution, other numerical methods [67] and present method in case of shear stress $\tau_{x y}$ along $x=24$ for this problem with 120 boundary nodes. The results show an excellent agreement between the exact solution and present method. 


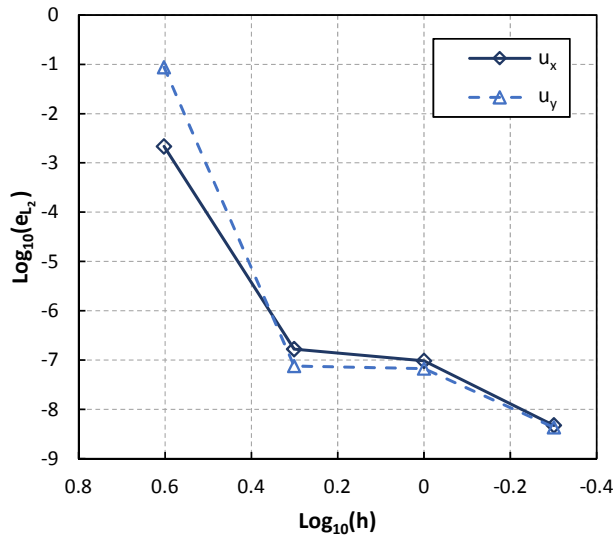

(a)

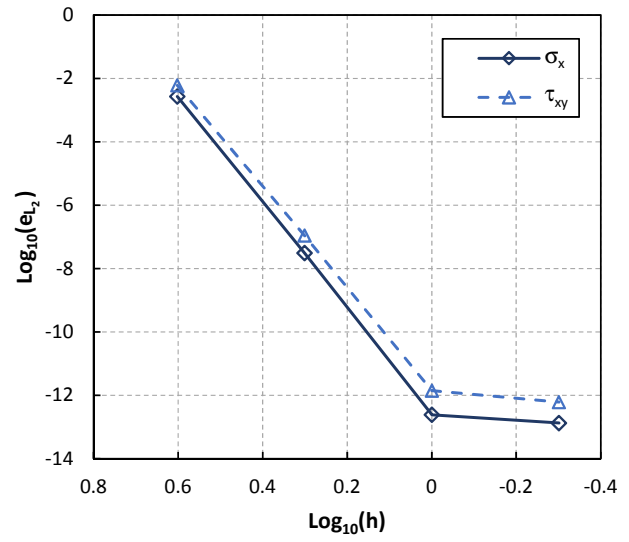

(b)

Figure 2. Convergence plot for Example 1: (a) displacements and (b) stresses

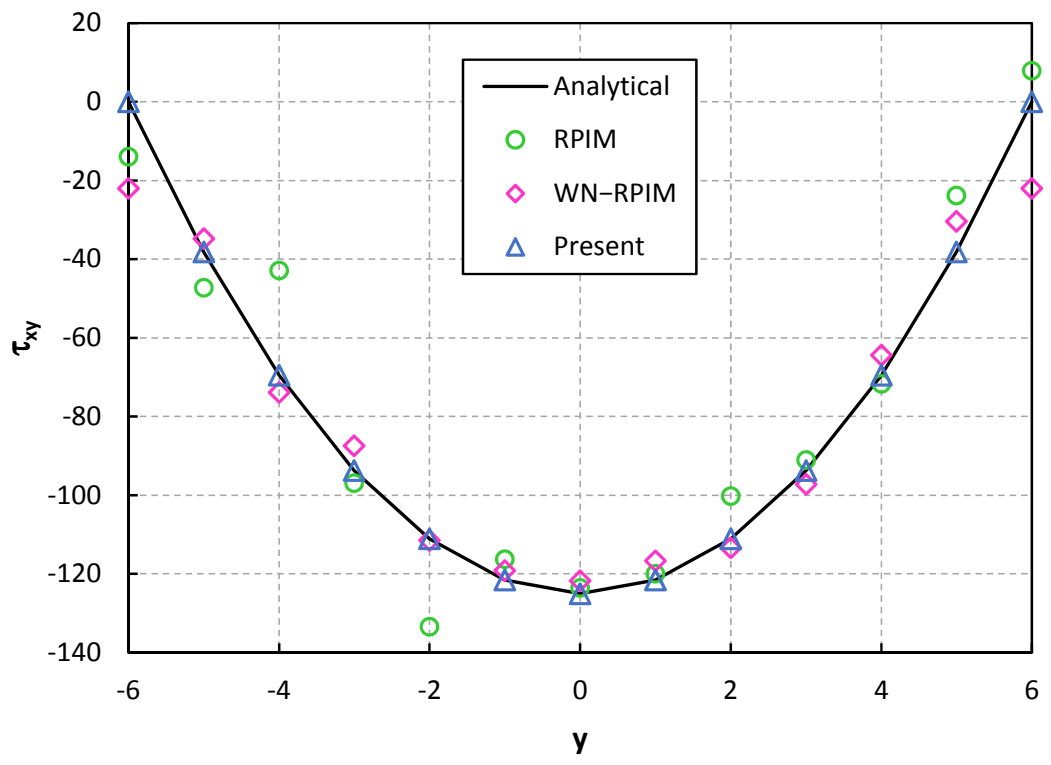

Figure 3. Comparison the shear stress distribution with 120 boundary nodes along $x=24$ for Example 1

\section{Example 2}

In this example we consider a non-homogeneous Helmholtz equation with variable coefficients in a square domain, namely

$$
\frac{\partial}{\partial x}\left(a(\mathbf{x}) \frac{\partial u}{\partial x}\right)+\frac{\partial}{\partial y}\left(a(\mathbf{x}) \frac{\partial u}{\partial y}\right)+k(\mathbf{x}) u=f
$$

where $a(\mathbf{x})$ and $k(\mathbf{x})$ are known variable material coefficient and known variable wave number, respectively. Taking these material parameters as

$$
a(\mathbf{x})=\exp (x+y), \quad k(\mathbf{x})=\sin x+\sin y
$$




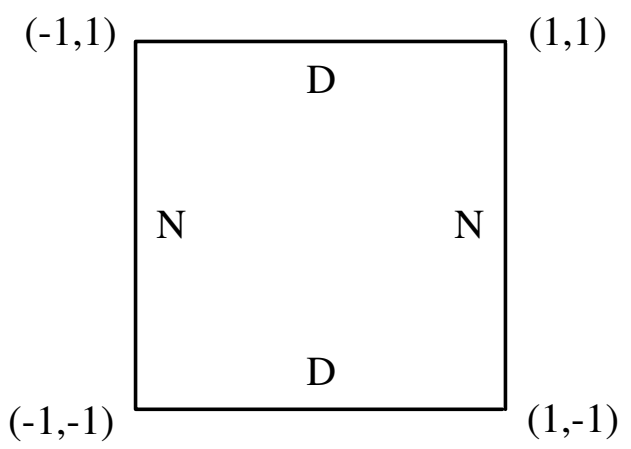

Figure 4. The geometry and boundary conditions of Example 2; 'D' and 'N' stand for Dirichlet and Neumann boundaries respectively

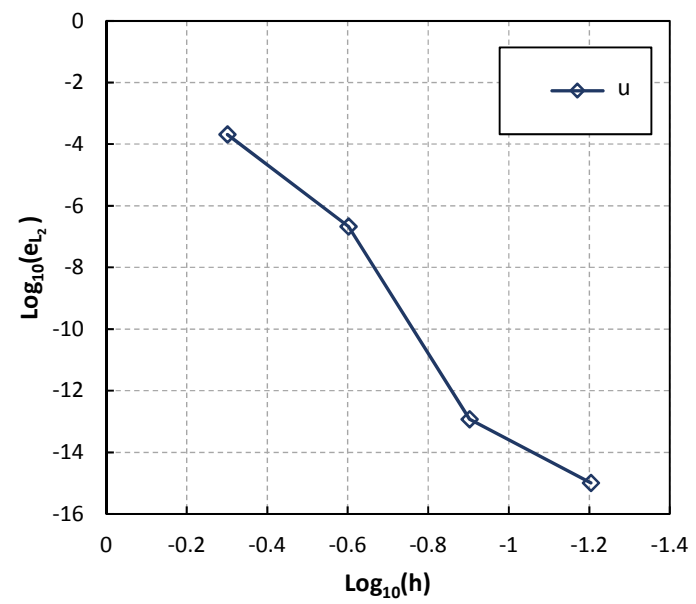

Figure 5. Convergence plot for non-homogeneous Helmholtz equation of Example 2

the analytical solution of this problem available in [68] will be

$$
u=x^{2}+y^{2}
$$

The problem domain and the defined boundary conditions for this example are illustrated in Figure 4. The source term $f$ and the boundary conditions are determined from the analytical solution. The convergence of the solution is shown in Figure 5. For a quantitative comparison, we used the results from various boundary element methods in [68]. Table I demonstrates the computed values of $u$ along the middle line of the domain using boundary-domain integro-differential equation (BDIDE), radial integration boundary integro-differential equation (RIBIDE), boundarydomain integral equation (BDIE), radial integration boundary integral equation (RIBIE), as well as analytical solution and present method. The results show an excellent agreement between the proposed method and the exact solution. 
Table I. Computed values of $u$ with 32 boundary nodes along line of $y=1.5$ in Example 2

\begin{tabular}{ccccccc}
\hline$x$ & BDIDE & RIBIDE & BDIE & RIBIE & GEBF & Exact \\
\hline 1.000 & 3.23907298 & 3.32246311 & 3.24875134 & 3.24996383 & 3.25000000 & 3.25000000 \\
1.125 & 3.50510973 & 3.57286266 & 3.51593634 & 3.51652295 & 3.51562500 & 3.51562500 \\
1.250 & 3.80153097 & 3.85513479 & 3.81314959 & 3.81320922 & 3.81249999 & 3.81250000 \\
1.375 & 4.12948095 & 4.17133413 & 4.14165412 & 4.14125138 & 4.14062499 & 4.14062500 \\
1.500 & 4.48895221 & 4.51957143 & 4.50149612 & 4.50063760 & 4.49999999 & 4.50000000 \\
1.625 & 4.87992777 & 4.89980552 & 4.89272494 & 4.89135856 & 4.89062499 & 4.89062500 \\
1.750 & 5.30237464 & 5.31223044 & 5.31538789 & 5.31340484 & 5.31249999 & 5.31250000 \\
1.875 & 5.75625542 & 5.75675965 & 5.76951472 & 5.76675756 & 5.76562499 & 5.76562500 \\
2.000 & 6.24013358 & 6.23692919 & 6.25374388 & 6.25000002 & 6.24999999 & 6.25000000 \\
\hline
\end{tabular}

\section{Example 3}

In this example we aim at employing the proposed method in solution of the Motz problem. The problem was first introduced by Motz [69]. Since then, many researchers selected it as a prototype of singular problems to verify the efficiency of numerical methods. The governing equation is the Laplace equation on a rectangular domain with the mixed Dirichlet/Neumann boundary conditions (viz. Figure 6) where, there is a sudden change in the boundary conditions at the origin. The asymptotic solution of this problem can be found in $[35,56]$ as

$$
u(r, \theta)=\sum_{i=0}^{\infty} d_{i} r^{i+1 / 2} \cos (i+1 / 2) \theta
$$

where $d_{i}$ are the expansion coefficients, and $(r, \theta)$ are the polar coordinates with the origin at $(0,0)$. In numerical solutions, one may use the above expansion with finite terms as

$$
u^{*}(r, \theta)=\sum_{i=0}^{n_{s}} D_{i} r^{i+1 / 2} \cos (i+1 / 2) \theta
$$

In this regard, to elucidate the accuracy of the present method for this problem, we use the $D_{i}$ coefficients reported in [56] for $n_{s}=34$. Due to the smooth and global nature of basis functions used in the proposed method, efficiency of the proposed method can be decreased in problems with singularities. However, we address this issue by adding a few singular functions to original bases (see [59] for more information). This has been done for a variety of methods, including the collocation Trefftz method (CTM) [35,56] and EBF itself [59]. Figure 7 illustrates the results obtained. Figure 8 indicates the error norm variation versus the number of degrees of freedom and the number of the singular bases. To get more insight into the performance of the proposed method in satisfaction of the boundary conditions, Figure 9 presents the errors on Neumann $(y=1,-1 \leq x \leq 1)$ and Dirichlet $(x=1,0 \leq y \leq 1)$ boundaries, respectively. It can be concluded that boundary conditions are satisfied accurately in the proposed method. 


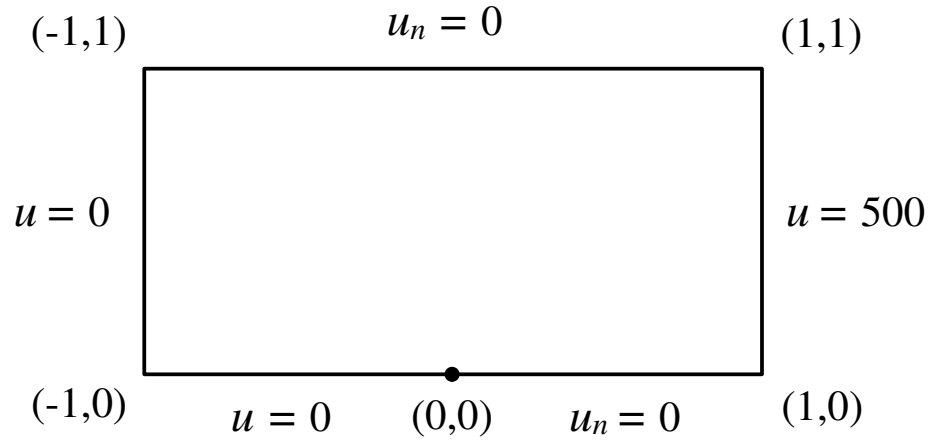

Figure 6. The geometry of Motz problem; $u$ and $u_{n}$ are prescribed values on Dirichlet and Neumann boundaries respectively

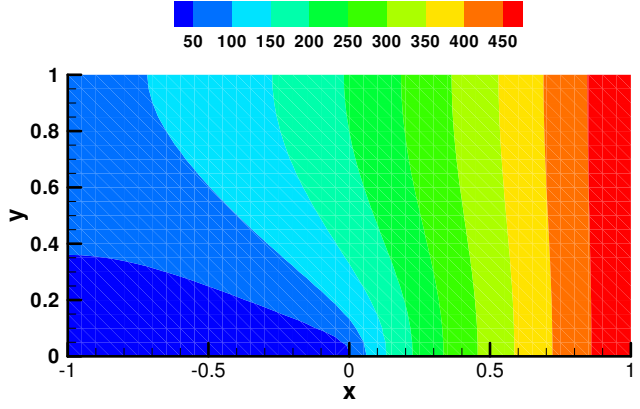

(a)

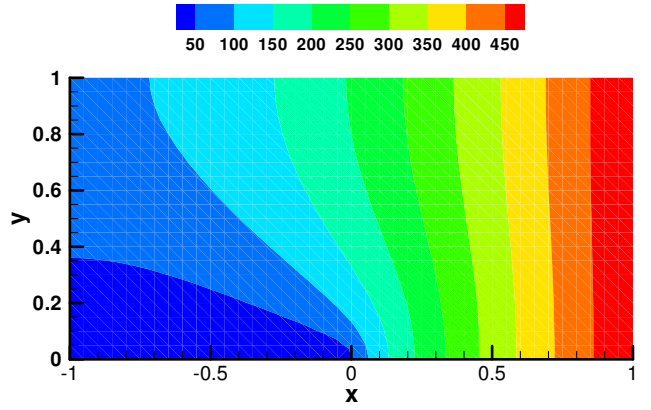

(b)

Figure 7. Contour plot of (a) numerical solution with 119 boundary nodes and (b) exact solution for Motz problem

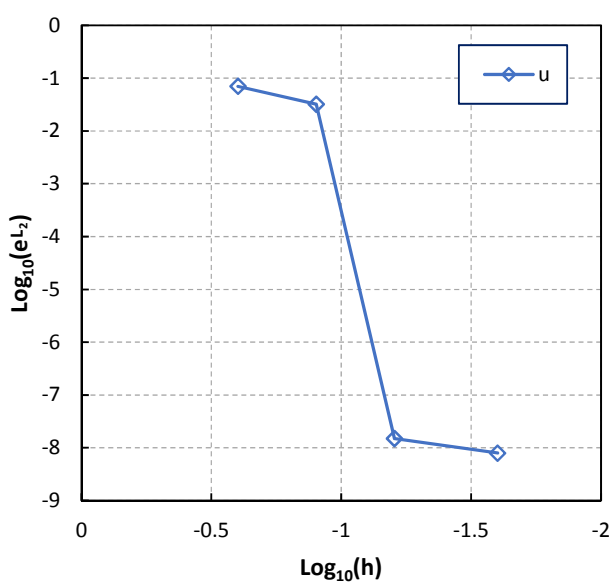

(a)

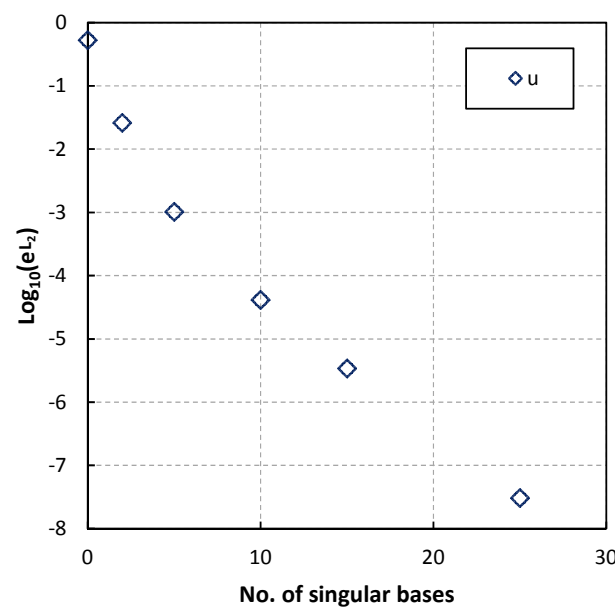

(b)

Figure 8. Convergence plot for the Motz problem of Example 3 based on; (a) average distance of nodes with 50 singular bases, (b) number of singular bases with $h=0.05$

\section{Example 4}

In order to test the method in the solution of non-linear PDEs, we consider a generalized non-linear Poisson problem in the square domain whose governing equation is defined by

Copyright (c) 0000 John Wiley \& Sons, Ltd. $\frac{\partial^{2} u}{\partial x^{2}}+\frac{\partial^{2} u}{\partial y^{2}}+\left(\frac{\partial u}{\partial y}\right)^{2}=f$
Prepared using nmeauth.cls

Int. J. Numer. Meth. Engng (0000) DOI: 10.1002 (24 489 


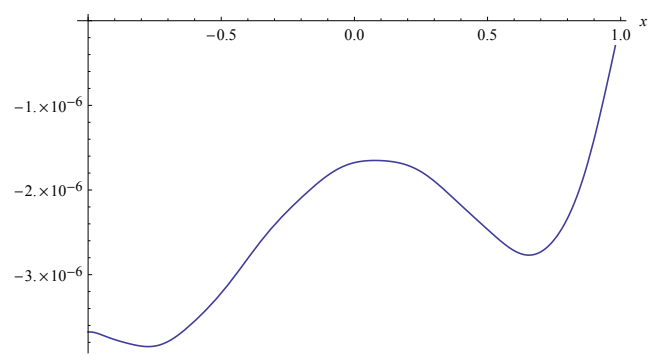

(a)

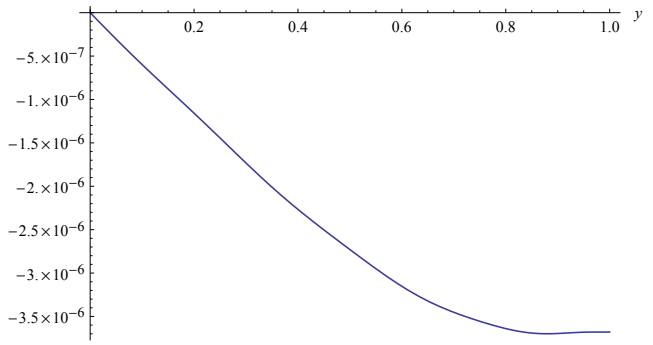

(b)

Figure 9. The boundary conditions satisfaction error in the Motz problem with 119 boundary nodes; (a) Neumann boundary $(y=1,-1 \leq x \leq 1)$ (b) Dirichlet boundary $(x=1,0 \leq y \leq 1)$

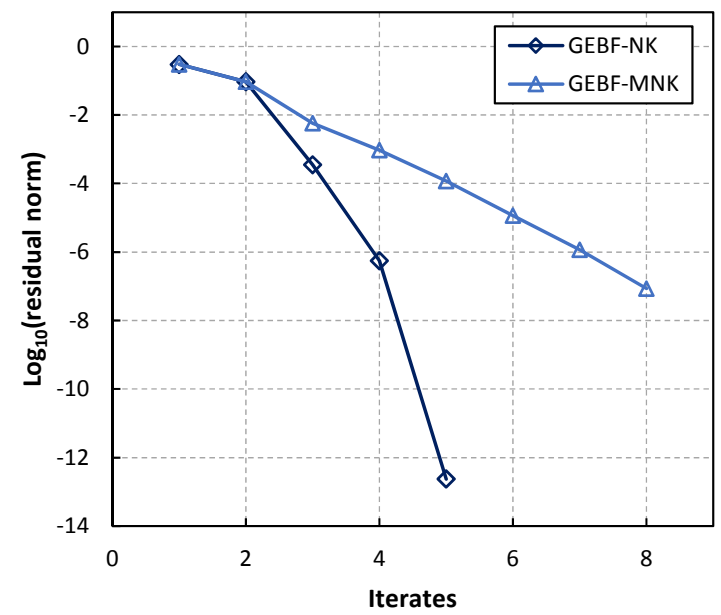

Figure 10. Convergence plot for generalized non-linear Poisson problem of Example 4

The exact solution of this problem can be found in [70] as

$$
u=x^{2} y
$$

The boundary conditions are assumed to be of Dirichlet type on all edges. The source term $f$ and the boundary conditions are obtained from the exact solution. Here, both NK and MNK methods are employed with a zero initial guess. The convergence history of the solution with 80 boundary nodes is shown in Figure 10. Figure 11 demonstrates the contour plot of the numerical results obtained from proposed method and the exact solution. Again, for a quantitative comparison we used the virtual boundary collocation method results discussed in [70]. Table II demonstrates the computed values of $u$ in various points of the domain, from the virtual boundary collocation method (VCBM), the analytical solution and the present method using NK approach.

\section{Example 5}

In this example we solve a 2D steady-state heat conduction problem over a non-homogeneous media. To this end, we consider a single anisotropic material in the square domain $[-1,1] \times[-1,1]$ representing a non-linear FGM with exponential heterogeneity. This problem is discussed in $[71,72]$ 


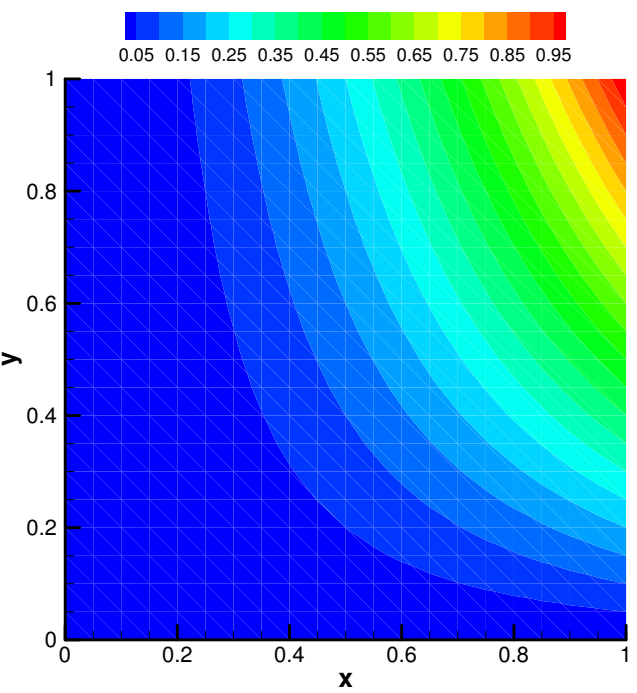

(a)

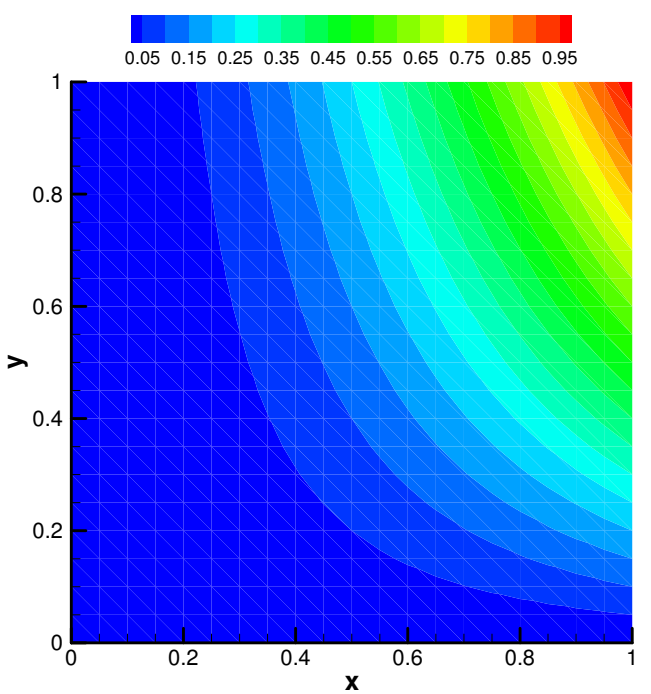

(b)

Figure 11. Contour plot of (a) numerical solution with 16 boundary nodes and (b) exact solution for Example 4

Table II. Comparison of numerical results for Example 4 with 32 boundary nodes

\begin{tabular}{cccc}
\hline$(x, y)$ & VBCM & GEBF $(\mathrm{NK})$ & Exact \\
\hline$(0.00,0.00)$ & 0.0000 & 0.000000 & 0.000000 \\
$(0.25,0.25)$ & 0.0159 & 0.015642 & 0.015625 \\
$(0.50,0.50)$ & 0.1251 & 0.124996 & 0.125000 \\
$(0.75,0.75)$ & 0.4222 & 0.421847 & 0.421875 \\
$(1.00,1.00)$ & 1.0000 & 1.000000 & 1.000000 \\
$(1,00,0.50)$ & 0.5000 & 0.500000 & 0.500000 \\
\hline
\end{tabular}

with heat conduction coefficients varying as functions of the temperature inside the material. The governing equation for steady-state heat conduction in heterogeneous media is defined by

$$
\nabla \cdot(\mathbf{K} \nabla T)=0
$$

where $\mathbf{K}$ denotes the thermal conductivity matrix. A FGM is supposed to make a composite material by varying the microstructure from one material to another with a specific gradient. In practice, FGMs usually serve in the high-temperature environments. Therefore, we consider the thermal conductivity as a non-linear function of temperature, such as

$$
\alpha(T)=\exp (T)
$$

To cope with non-linear behavior of the above term, the Kirchhoff transformation is employed (see [73] for more information). The thermal conductivity matrix $\mathbf{K}$ defined as

$$
\left(\begin{array}{cc}
k_{1} & 0 \\
0 & k_{2}
\end{array}\right)=\sqrt{\frac{1-c / r}{2 r}} \sinh (r) \exp (y)\left(\begin{array}{ll}
2 & 0 \\
0 & 1
\end{array}\right)
$$




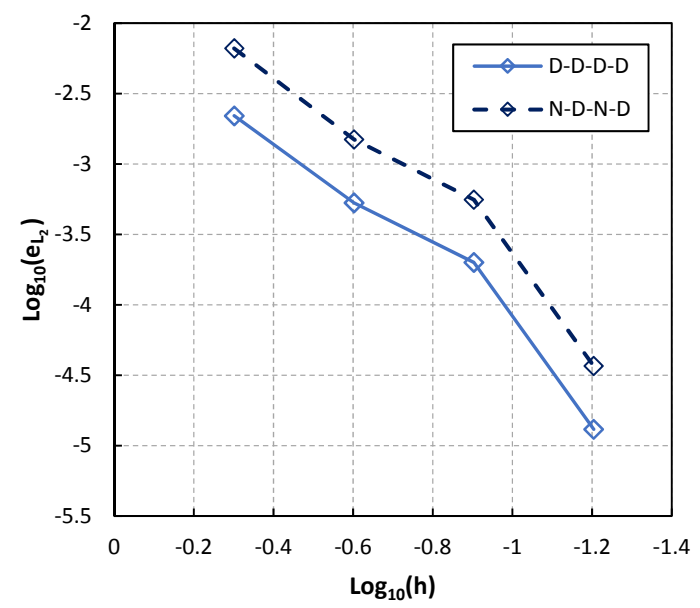

Figure 12. Convergence plot of steady-state heat conduction problem in Example 5

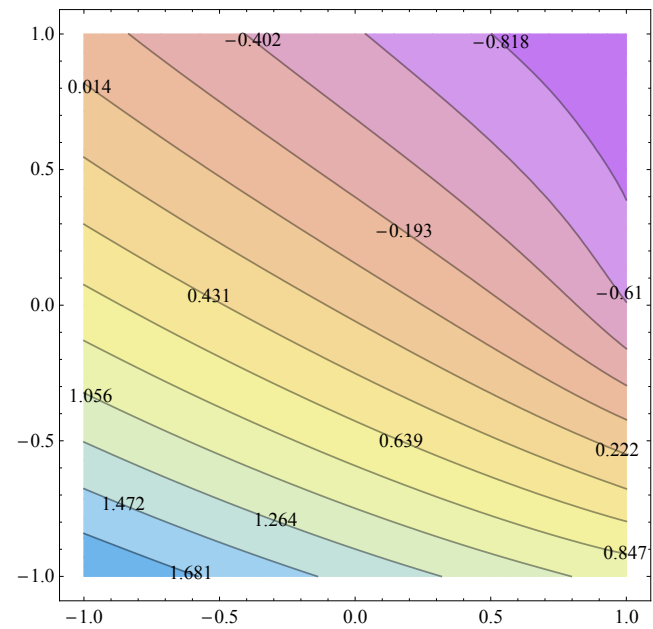

(a)

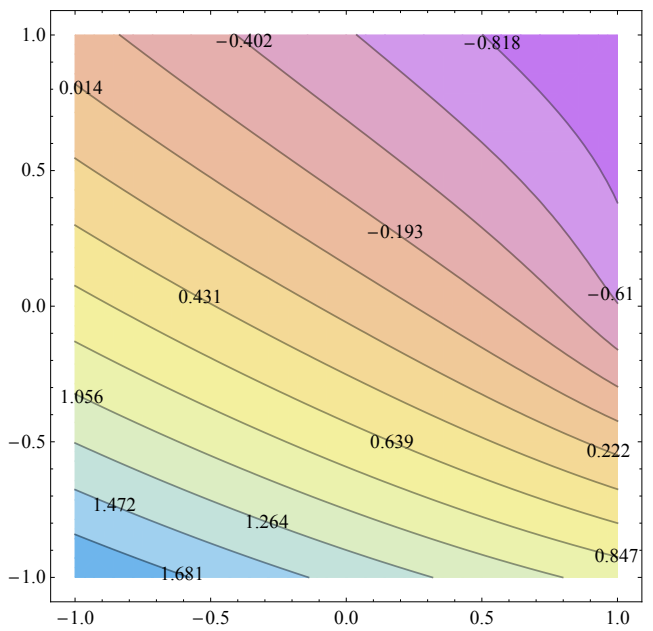

(b)

Figure 13. The isothermals throughout the FGM in Example 5 (a) from the numerical solution with 32 boundary nodes and (b) from the analytical solution

where $c$ and $r$ are taken as $x / \sqrt{2}-1$ and $\sqrt{c^{2}+y^{2}}$, respectively. The analytical solution for this problem is expressed as

$$
T=\ln \left(\sqrt{\frac{1-c / r}{2 r}} \sinh (r) \exp (-y)\right)
$$

The problem has been solved with two types of boundary conditions. First, the boundary conditions are assumed to be of Dirichlet type on all edges (D-D-D-D). Again, the mixed Dirichlet/Neumann boundary conditions (N-D-N-D) are considered on boundaries. Figure 12 shows the convergence of the solution. Figure 13 illustrates isothermal contours of the FGM plate under Dirichlet boundary conditions with 32 boundary nodes. It can be seen from Figure 13 that the numerical solution is in excellent agreement with the analytical solution. 


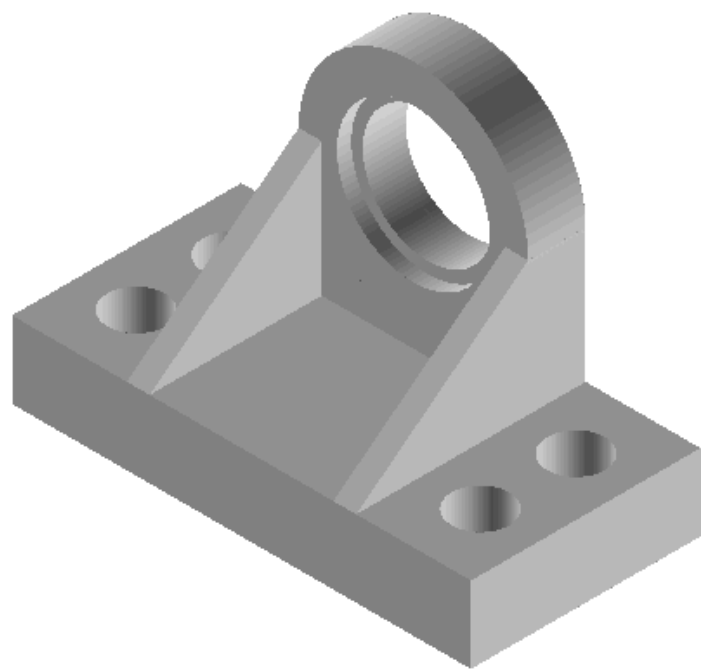

Figure 14. A 3D axletree base

\section{Example 6}

In this example the proposed method is applied to solve a 3D problem. Three-dimensional problems are usually not easy to deal with partly due to the high effort in the mesh generation for meshdependent methods such as FEM. The goal of the following experiment is to demonstrate the accuracy and efficiency of the present method to solve 3D problems. We study the heat conduction over a 3D axletree base. The geometry of this problem is depicted in Figure 14. The 3D axletree base has also been used previously in [74], and is regenerated here. The governing equation for this example given by (50). Here, we consider the homogenous and isotropic steady-state characteristics of heat conduction with given thermal conductivity matrix $\mathbf{K}$ as an identity matrix. The problem has been solved under Dirichlet boundary conditions. The exact solution for this example is chosen as a 3D harmonic polynomial as

$$
\begin{aligned}
T= & x^{10}+10 x^{9} y+45 x^{8} y^{2}-90 x^{8} z^{2}+120 x^{7} y^{3}-720 x^{7} y z^{2}+210 x^{6} y^{4}-2520 x^{6} y^{2} z^{2}+ \\
& 840 x^{6} z^{4}+252 x^{5} y^{5}-5040 x^{5} y^{3} z^{2}+5040 x^{5} y z^{4}+210 x^{4} y^{6}-6300 x^{4} y^{4} z^{2}+ \\
& 12600 x^{4} y^{2} z^{4}-1680 x^{4} z^{6}+120 x^{3} y^{7}-5040 x^{3} y^{5} z^{2}+16800 x^{3} y^{3} z^{4}-6720 x^{3} y z^{6}+ \\
& 45 x^{2} y^{8}-2520 x^{2} y^{6} z^{2}+12600 x^{2} y^{4} z^{4}-10080 x^{2} y^{2} z^{6}+720 x^{2} z^{8}+10 x y^{9}- \\
& 720 x y^{7} z^{2}+5040 x y^{5} z^{4}-6720 x y^{3} z^{6}+1440 x y z^{8}+y^{10}-90 y^{8} z^{2}+840 y^{6} z^{4}- \\
& 1680 y^{4} z^{6}+720 y^{2} z^{8}-32 z^{10}
\end{aligned}
$$

Table III demonstrates the numerical results obtained along with other assumptions. The numerical results show the desirable performance of the present method to deal with the three-dimensional problems. 
Table III. The results obtained for Example 6

\begin{tabular}{ccccc}
\hline Domain nodes $\left(n_{\Omega}\right)$ & Boundary nodes $\left(n_{\Gamma}\right)$ & Bases $(m)$ & Null space bases $(b)$ & $e_{L_{2}}$ \\
\hline 751 & 614 & 1458 & 848 & $5.27405 \times 10^{-3}$ \\
751 & 614 & 8192 & 7441 & $4.83870 \times 10^{-9}$ \\
\hline
\end{tabular}

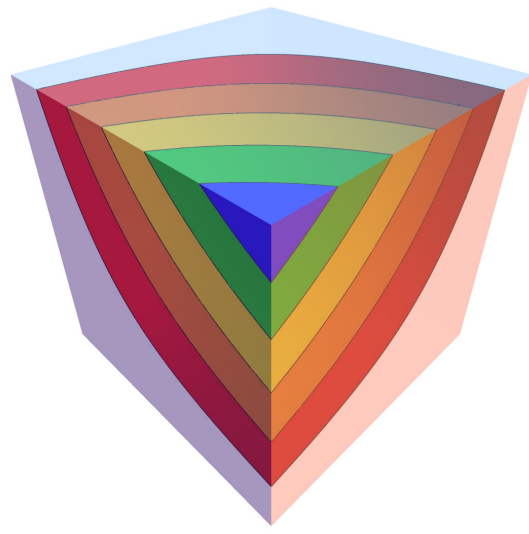

(a)

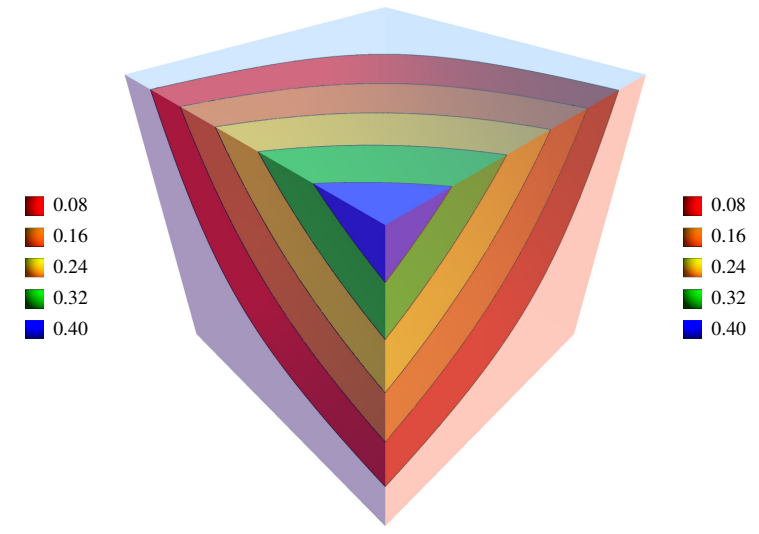

(b)

Figure 15. Contour plot of (a) numerical solution and (b) exact solution for Example 7

Table IV. Total CPU time spent for obtaining the numerical results of the same accuracy in $e_{L_{2}} \cong 1.2 \times 10^{-7}$

\begin{tabular}{ccc}
\hline Methods & Total CPU time $(\mathrm{s})$ & Ratio \\
\hline FEM & 65.69 & 1 \\
GEBF & 0.39 & $1 / 167.59$ \\
\hline
\end{tabular}

\section{Example 7}

Finally, to demonstrate the relative computational cost of the present method, we solve a Laplace equation over a cube with unit side length. The governing equation for this is defined as

$$
\nabla \cdot \nabla u=0
$$

The exact solution to this problem is taken to be

$$
u=\sin \left(\frac{3}{5} x\right) \sin \left(\frac{4}{5} y\right) \sinh (z)
$$

Dirichlet boundary conditions are assumed on all sides of the cube. Figure 15 demonstrates the contour plot of the numerical results obtained from proposed method along with the exact solution. We compare the run time of the proposed method with that of Kratos, a parallel, highly optimized finite element code [54]. In Kratos we employ a regular mesh of 8 node linear brick elements. The tests are carried out on a Intel Core i7 2700K CPU at 2.80GHz with 8 GB of DDR3 RAM running Ubuntu 14.0464 bit. We refined the mesh in the FEM until almost the same level of error $e_{L_{2}} \cong 1.2 \times 10^{-7}$ earned in both methods. Total CPU time spent are reported in Table IV. It is found that the GEBF in this specific case is more efficient than the FEM by a factor of 167. 


\section{CONCLUSION}

A generalized exponential basis functions method was proposed. The proposed method addresses the shortcomings of the original exponential basis function method by leveraging the method to general linear and non-linear problems. A Newton-Kantorovich scheme used in non-linear problems to establish an iterative, linear set of equations which is solved using the proposed method in linear problems. Details of an efficient implementation is discussed. The method is shown to perform well in some benchmark problems, compared to a variety of the methods in the literature. Also, we show that the proposed method can solve 3D problems with complex geometries. The performance of the proposed method is compared to a parallel, highly optimized finite element code, Kratos. The method fully shows its potential when applied to 3D problems. For the test case considered, which represents a simple yet representative benchmark case, the proposed method is over 167 times more efficient, when the same level of error is targeted. Similar gains can be expected in other problems of similar characteristics, where the enhanced properties of the GEBF allow reaching the same level of accuracy as the FEM using a much lower degrees of freedom. Practical limits might be found however for large problems because of dense matrix operations involved. It is noted that domain decomposition and local schemes are possible and the method can be extended to large deformation and materially non-linear cases in the future. 3D singular problems is another topic which may be investigated, taking into account the characteristics of the proposed method.

\section{REFERENCES}

1. Lucy LB. A numerical approach to the testing of the fission hypothesis. The astronomical journal 1977; 82:10131024.

2. Gingold RA, Monaghan JJ. Smoothed particle hydrodynamics: theory and application to non-spherical stars. Monthly notices of the royal astronomical society 1977; 181(3):375-389.

3. Belytschko T, Lu YY, Gu L. Elementfree Galerkin methods. International journal for numerical methods in engineering 1994; 37(2):229-256.

4. Atluri SN, Zhu T. A new meshless local Petrov-Galerkin (MLPG) approach in computational mechanics. Computational mechanics 1998; 22(2):117-127.

5. Oñate E, Idelsohn S, Zienkiewicz OC, Taylor RL. A finite point method in computational mechanics. Applications to convective transport and fluid flow. International journal for numerical methods in engineering 1996; 39(22):3839-3866.

6. Oñate E, Idelsohn S, Zienkiewicz OC, Taylor RL, Sacco C. A stabilized finite point method for analysis of fluid mechanics problems. Computer Methods in Applied Mechanics and Engineering 1996; 139(1):315-346.

7. Oñate E, Perazzo F, Miquel J. A finite point method for elasticity problems. Computers \& Structures 2001; 79(22):2151-2163.

8. Löhner R, Sacco C, Oñate E, Idelsohn S. A finite point method for compressible flow. International journal for numerical methods in engineering 2002; 53(8):1765-1779.

9. Kansa EJ. Multiquadricsa scattered data approximation scheme with applications to computational fluid-dynamicsI surface approximations and partial derivative estimates. Computers \& mathematics with applications 1990; 19(8):127-145.

10. Kansa EJ. MultiquadricsA scattered data approximation scheme with applications to computational fluiddynamicsII solutions to parabolic, hyperbolic and elliptic partial differential equations. Computers \& mathematics with applications 1990; 19(8):147-161.

11. Franke C, Schaback R. Solving partial differential equations by collocation using radial basis functions. Applied Mathematics and Computation 1998; 93(1):73-82.

12. Zhang X, Song KZ, Lu MW, Liu X. Meshless methods based on collocation with radial basis functions. Computational mechanics 2000; 26(4):333-343. 
13. Mai-Duy N, Tran-Cong T. Numerical solution of differential equations using multiquadric radial basis function networks. Neural Networks 2001; 14(2):185-199.

14. Larsson E, Fornberg B. A numerical study of some radial basis function based solution methods for elliptic PDEs. Computers \& mathematics with applications 2003; 46(5):891-902.

15. Buhmann MD. Radial basis functions: Theory and implementations, vol. 5. Cambridge university press Cambridge, 2003.

16. Ingber MS, Chen CS, Tanski JA. A mesh free approach using radial basis functions and parallel domain decomposition for solving threedimensional diffusion equations. International journal for numerical methods in engineering 2004; 60(13):2183-2201.

17. Šarler B, Vertnik R. Meshfree explicit local radial basis function collocation method for diffusion problems. Computers \& mathematics with applications 2006; 51(8):1269-1282.

18. Vertnik R, Šarler B. Meshless local radial basis function collocation method for convective-diffusive solid-liquid phase change problems. International Journal of Numerical Methods for Heat \& Fluid Flow 2006; 16(5):617-640.

19. Fairweather G, Karageorghis A. The method of fundamental solutions for elliptic boundary value problems. Advances in Computational Mathematics 1998; 9(1-2):69-95.

20. Karageorghis A, Fairweather G. The method of fundamental solutions for axisymmetric potential problems. International journal for numerical methods in engineering 1999; 44(11):1653-1669.

21. Ramachandran PA. Method of fundamental solutions: singular value decomposition analysis. Communications in numerical methods in engineering 2002; 18(11):789-801.

22. Chen CW, Young DL, Tsai CC, Murugesan K. The method of fundamental solutions for inverse 2D Stokes problems. Computational mechanics 2005; 37(1):2-14.

23. Chantasiriwan S. Methods of fundamental solutions for timedependent heat conduction problems. International journal for numerical methods in engineering 2006; 66(1):147-165.

24. Jin B, Zheng Y, Marin L. The method of fundamental solutions for inverse boundary value problems associated with the steadystate heat conduction in anisotropic media. International journal for numerical methods in engineering 2006; 65(11):1865-1891.

25. Wei T, Hon YC, Ling L. Method of fundamental solutions with regularization techniques for Cauchy problems of elliptic operators. Engineering Analysis with Boundary Elements 2007; 31(4):373-385.

26. Steinbach O. Numerical approximation methods for elliptic boundary value problems 2008.

27. Alves CJS, Antunes PRS. The method of fundamental solutions applied to the calculation of eigensolutions for 2D plates. International journal for numerical methods in engineering 2009; 77(2):177-194.

28. Trefftz E. Ein gegenstuck zum ritzschen verfahren. Proceedings of the 2nd International Congress for Applied Mechanics 1926; :131-137.

29. Zieliski AP, Herrera I. Trefftz method: fitting boundary conditions. International journal for numerical methods in engineering 1987; 24(5):871-891.

30. Zieliski AP. Trefftz method: elastic and elastoplastic problems. Computer Methods in Applied Mechanics and Engineering 1988; 69(2):185-204.

31. Cheung YK, Jin WG, Zienkiewicz OC. Direct solution procedure for solution of harmonic problems using complete, nonsingular, Trefftz functions. Communications in applied numerical methods 1989; 5(3):159-169.

32. Jin WG, Cheung YK, Zienkiewicz OC. Application of the Trefftz method in plane elasticity problems. International journal for numerical methods in engineering 1990; 30(6):1147-1161.

33. Cheung YK, Jin WG, Zienkiewicz OC. Solution of Helmholtz equation by Trefftz method. International journal for numerical methods in engineering 1991; 32(1):63-78.

34. Kita E, Kamiya N. Trefftz method: an overview. Advances in Engineering Software 1995; 24(1):3-12.

35. Li ZC, Lu TT, Hu HY, Cheng AHD. Trefftz and collocation methods. WIT Press Southampton, Boston, 2008.

36. Boroomand B, Soghrati S, Movahedian B. Exponential basis functions in solution of static and time harmonic elastic problems in a meshless style. International Journal for Numerical Methods in Engineering 2010; 81(8):9711018.

37. Shahbazi M, Boroomand B, Soghrati S. A mesh-free method using exponential basis functions for laminates modeled by CLPT, FSDT and TSDTPart II: Implementation and results. Composite Structures 2011; 94(1):8491.

38. Shahbazi M, Boroomand B, Soghrati S. A mesh-free method using exponential basis functions for laminates modeled by CLPT, FSDT and TSDTPart I: Formulation. Composite Structures 2011; 93(12):3112-3119.

39. Shamsaei B, Boroomand B. Exponential basis functions in solution of laminated structures. Composite Structures 2011; 93(8):2010-2019.

40. Shahbazi M, Boroomand B, Soghrati S. On using exponential basis functions for laminates modeled by CLPT, FSDT and TSDT: further tests and results. Composite Structures 2012; 94(7):2263-2268. 
41. Zandi SM, Boroomand B, Soghrati S. Exponential basis functions in solution of problems with fully incompressible materials: A mesh-free method. Journal of Computational Physics 2012; 231(21):7255-7273.

42. Zandi SM, Boroomand B, Soghrati S. Exponential basis functions in solution of incompressible fluid problems with moving free surfaces. Journal of Computational Physics 2012; 231(2):505-527.

43. Azhari F, Boroomand B, Shahbazi M. Explicit relations for the solution of laminated plates modeled by a higher shear deformation theory: Derivation of exponential basis functions. International Journal of Mechanical Sciences 2013; 77:301-313.

44. Azhari F, Boroomand B, Shahbazi M. Exponential basis functions in the solution of laminated plates using a higherorder ZigZag theory. Composite Structures 2013; 105:398-407.

45. Boroomand B, Azhari F, Shahbazi M. On definition of clamped conditions in TSDT and FSDT; the use of exponential basis functions in solution of laminated composites. Composite Structures 2013; 97:129-135.

46. Hashemi SH, Boroomand B, Movahedian B. Exponential basis functions in space and time: A meshless method for 2D time dependent problems. Journal of Computational Physics 2013; 241:526-545.

47. Movahedian B, Boroomand B, Soghrati S. A Trefftz method in space and time using exponential basis functions: Application to direct and inverse heat conduction problems. Engineering Analysis with Boundary Elements 2013; 37(5):868-883.

48. Movahedian B, Boroomand B. The solution of direct and inverse transient heat conduction problems with layered materials using exponential basis functions. International Journal of Thermal Sciences 2014; 77:186-198.

49. Abdollahi R, Boroomand B. Nonlocal elasticity defined by Eringens integral model: Introduction of a boundary layer method. International journal of solids and structures 2014; 51(9):1758-1780.

50. Soleimanifar E, Boroomand B, Mossaiby F. A meshless method using local exponential basis functions with weak continuity up to a desired order. Computational Mechanics 2014; 53(6):1355-1374.

51. Shojaei A, Boroomand B, Mossaiby F. A simple meshless method for challenging engineering problems. Engineering Computations 2015; Accepted.

52. Boroomand B, Noormohammadi N. Weakly equilibrated basis functions for elasticity problems. Engineering Analysis with Boundary Elements 2013; 37(12):1712-1727.

53. Noormohammadi N, Boroomand B. A fictitious domain method using equilibrated basis functions for harmonic and bi-harmonic problems in physics. Journal of Computational Physics Sep 2014; 272:189-217.

54. Dadvand P, Rossi R, Oñate E. An Object-oriented Environment for Developing Finite Element Codes for Multidisciplinary Applications. Archives of Computational Methods in Engineering Jul 2010; 17(3):253-297, doi: 10.1007/s11831-010-9045-2.

55. Young DL, Fan CM, Tsai CC, Chen CW. The method of fundamental solutions and domain decomposition method for degenerate seepage flownet problems. Journal of the Chinese Institute of Engineers Jan 2006; 29(1):63-73, doi:10.1080/02533839.2006.9671099.

56. Lu TT, Hu HY, Li ZC. Highly accurate solutions of Motz's and the cracked beam problems. Engineering Analysis with Boundary Elements 2004; 28(11):1387-1403.

57. Li Z, Lu T, Huang H, Cheng AH. Trefftz, collocation, and other boundary methodsa comparison. Numerical Methods for Partial Differential Equations 2007; 23(1):93-144.

58. Li Z, Lee M, Chiang JY. Collocation Trefftz methods for the stokes equations with singularity. Numerical Methods for Partial Differential Equations 2013; 29(2):361-395.

59. Mossaiby F, Bazrpach M, Shojaei A. Extending the method of exponential basis functions to problems with singularities. Engineering Computations 2014; Accepted.

60. Argyros IK. Convergence and applications of Newton-type iterations, vol. 337. Springer, 2008.

61. Ben-Israel A, Greville TNE. Generalized inverses, vol. 13. Springer, 2003.

62. Linear algebra package (lapack). URL http://net lib.org/lapack.

63. Openblas. URL http: //www. openblas. net.

64. Basic linear algebra subprograms (blas). URL http://netlib.org/blas.

65. Generalized exponential basis functions (gebf) method. URL https ://bitbucket.org/mossaiby/gebf.

66. Timoshenko SP, Goodier JN, Abramson HN. Theory of elasticity. McGraw-Hill: New York, 1970.

67. Cao Y, Yao LQ, Yi SC. A weighted nodal-radial point interpolation meshless method for 2D solid problems. Engineering Analysis with Boundary Elements 2014; 39:88-100.

68. Al-Jawary MA, Wrobel LC. Numerical solution of the two-dimensional Helmholtz equation with variable coefficients by the radial integration boundary integral and integro-differential equation methods. International Journal of Computer Mathematics 2012; 89(11):1463-1487.

69. Motz H. The treatment of singularities of partial differential equations by relaxation methods. Quart. Appl. Math 1946; 4:371-377. 
70. Wang H, Qin QH. A meshless method for generalized linear or nonlinear Poisson-type problems. Engineering Analysis with Boundary Elements 2006; 30(6):515-521.

71. Marin L, Lesnic D. The method of fundamental solutions for nonlinear functionally graded materials. International journal of solids and structures 2007; 44(21):6878-6890.

72. Fu ZJ, Chen W, Qin QH. Boundary knot method for heat conduction in nonlinear functionally graded material. Engineering Analysis with Boundary Elements 2011; 35(5):729-734.

73. Kim S. A simple direct estimation of temperature-dependent thermal conductivity with Kirchhoff transformation. International communications in heat and mass transfer 2001; 28(4):537-544.

74. Liu GR. AG space theory and a weakened weak (W2) form for a unified formulation of compatible and incompatible methods: Part II applications to solid mechanics problems. International journal for numerical methods in engineering 2010; 81(9):1127-1156. 